\title{
Association between Plasma Sestrin2 Levels and the Presence and Severity of Coronary Artery Disease
}

\author{
Yoshimi Kishimoto, ${ }^{1}$ Masayuki Aoyama, ${ }^{2}$ Emi Saita, ${ }^{1}$ Yukinori Ikegami, ${ }^{2}$ Reiko Ohmori, ${ }^{3}$ \\ Kazuo Kondo, ${ }^{1,4}$ and Yukihiko Momiyama ${ }^{2}$ \\ ${ }^{1}$ Endowed Research Department "Food for Health", Ochanomizu University, Tokyo, Japan \\ ${ }^{2}$ Department of Cardiology, National Hospital Organization Tokyo Medical Center, Japan \\ ${ }^{3}$ Faculty of Regional Design, Utsunomiya University, Tochigi, Japan \\ ${ }^{4}$ Institute of Life Innovation Studies, Toyo University, Gunma, Japan
}

Correspondence should be addressed to Yukihiko Momiyama; ymomiyamajp@gmail.com

Received 19 December 2019; Revised 7 June 2020; Accepted 12 June 2020; Published 30 June 2020

Academic Editor: Benoit Dugue

Copyright (c) 2020 Yoshimi Kishimoto et al. This is an open access article distributed under the Creative Commons Attribution License, which permits unrestricted use, distribution, and reproduction in any medium, provided the original work is properly cited.

\begin{abstract}
Aims. Atherosclerotic disease, such as coronary artery disease (CAD), is recognized to be associated with inflammation and oxidative stress. We investigated the association between CAD and plasma levels of sestrin2 which is one of the stress-inducible antioxidant proteins. Methods. We measured plasma sestrin2 levels in 304 patients undergoing elective coronary angiography. The severity of CAD was represented as the numbers of $>50 \%$ stenotic coronary vessels and segments and the severity score. Results. CAD was found in 175 patients, of whom 73 had 1-vessel (1-VD), 59 had 2-vessel (2-VD), and 43 had 3-vessel disease (3-VD). Plasma sestrin2 levels were significantly higher in 175 patients with CAD than in 129 without CAD (median 16.4 vs. $14.2 \mathrm{ng} / \mathrm{mL}, P<0.05)$. A stepwise increase in sestrin2 levels was found depending on the number of $>50 \%$ stenotic coronary vessels: 14.2 in $\mathrm{CAD}(-), 15.4$ in $1-\mathrm{VD}, 17.3$ in $2-\mathrm{VD}$, and $17.7 \mathrm{ng} / \mathrm{mL}$ in3-VD $(P<0.05)$. High sestrin2 level $(>16.0 \mathrm{ng} / \mathrm{mL})$ was present in $38 \%$ of patients with $\mathrm{CAD}(-), 47 \%$ of $1-\mathrm{VD}, 66 \%$ of $2-\mathrm{VD}$, and $53 \%$ of $3-\mathrm{VD}(P<0.005)$. Sestrin2 levels significantly, but weakly, correlated with the number of $>50 \%$ stenotic segments and the severity score ( $\mathrm{rs}=0.12$ and $\mathrm{rs}=0.13, P<0.05$ ). In the multivariate analysis, sestrin2 levels were a significant factor associated with CAD independent of atherosclerotic risk factors. The odds ratio for CAD was $1.79(95 \% \mathrm{CI}=1.09-2.95)$ for high sestrin 2 level of $>16.0 \mathrm{ng} / \mathrm{mL}(P<0.025)$. Conclusions. Plasma sestrin2 levels in patients with CAD were found to be high and to be associated with the severity of CAD. High sestrin2 levels in patients with CAD may reflect a protective response against the progression of CAD.
\end{abstract}

\section{Introduction}

Atherosclerotic disease, such as coronary artery disease (CAD), is recognized to be a chronic inflammatory disease, and oxidative stress by excessive reactive oxygen species (ROS) contributes to the pathogenesis of atherosclerosis $[1,2]$. Endogenous antioxidants may play a protective role against the progression of atherosclerosis $[1,2]$. Sestrin2 is a stress-inducible antioxidant protein that was originally identified as a hypoxiainduced gene 95 (Hi95) [3]. Sestrin2 is expressed and secreted mainly by macrophages, $\mathrm{T}$ lymphocytes, and endothelial cells [4-6]. Sestrin2 expression was induced by angiotensin II in human umbilical vein endothelial cells (HUVECs), and the knockdown of sestrin 2 promoted angiotensin II-induced apoptosis and oxidative stress [7]. Sestrin2 expression was also upregulated in macrophages by oxidized LDL, and the upregulation of sestrin2 reduced cell apoptosis and oxidative stress [4]. Moreover, sestrin2 reduced lipopolysaccharide-induced proinflammatory cytokine release, cell death, and ROS production in macrophages [8]. The knockdown of sestrin2 in HUVECs promoted lipopolysaccharide-induced inflammatory responses, cell death, and ROS production [9]. These evidences thus suggest that sestrin 2 has antioxidant and anti-inflammatory properties. 
Atherosclerosis is recognized to be associated with inflammation with oxidative stress $[1,2]$, and sestrin 2 is suggested to play a protective role against atherosclerotic disease $[10,11]$. Regarding the association between sestrin 2 and atherosclerotic disease, one small study [12] recently reported that plasma sestrin2 levels were higher in 44 patients with stable CAD than in 35 patients without CAD. However, in their study, multivariate analysis was not performed in spite of significant differences in atherosclerotic risk factors between patients with and without CAD. In the present study, we investigated the associations between plasma sestrin2 levels and the presence and severity of CAD in 304 patients undergoing elective coronary angiography.

\section{Methods}

2.1. Study Patients. We measured plasma sestrin 2 levels in 304 consecutive patients who underwent elective coronary angiography for suspected CAD at Tokyo Medical Center from June 2009 to September 2016. Patients with acute coronary syndrome, defined as acute myocardial infarction (AMI) and class III unstable angina at rest by Braunwald's classification [13], were excluded from this study. Patients with a history of percutaneous coronary intervention or cardiac surgery or those with known aortic diseases were also excluded. Since plasma sestrin2 levels in patients with heart failure were reported to be high and to increase from New York Heart Association functional class II to IV [6], patients with a history of heart failure were also excluded from this study. Hypertension was defined as blood pressures of $\geq 140 / 90 \mathrm{mmHg}$ or on drugs, and 180 (59\%) patients were taking antihypertensive drugs. Hyperlipidemia was defined as an LDL-cholesterol level of $>140 \mathrm{mg} / \mathrm{dL}$ or on drugs, and $116(38 \%)$ patients were taking statins. Diabetes mellitus (DM) (a fasting plasma glucose (FPG) level of $\geq 126 \mathrm{mg} / \mathrm{dL}$ or on treatment) was present in $83(27 \%)$ patients, and 106 (35\%) were smokers ( $\geq 10$ pack-years). Our study was approved by the institutional ethics committee of our hospital (R07-054/R15-056). After written informed consent was obtained, overnight-fasting blood samples were taken on the morning of the day when coronary angiography was performed.

2.2. Measurements of Plasma Sestrin2 and C-reactive Protein (CRP) Levels. Blood samples were collected in EDTAcontaining tubes, and the plasma was stored at $-80^{\circ} \mathrm{C}$. Plasma sestrin2 levels were measured by an enzyme-linked immunosorbent assay (ELISA) with a commercially available kit (ELISA Kit for Sestrin2, Cloud-Clone, TX, USA) at Ochanomizu University according to the manufacturer's instructions. The intra- and interassay coefficients of variation were $<10 \%$ and $<12 \%$, respectively. Plasma high-sensitivity C-reactive protein (hsCRP) levels were also measured by a BNII nephelometer (Dade Behring, Tokyo, Japan).

2.3. Coronary Angiography. Coronary angiograms were recorded on a cineangiogram system (Philips Electronics Japan, Tokyo, Japan). CAD was defined as at least one coronary artery having $>50 \%$ luminal diameter stenosis on angio- grams. The severity of CAD was represented as the numbers of $>50 \%$ stenotic vessels and stenotic segments and the severity score of stenosis. The degree of coronary stenosis in each segment was scored from 0 to 4 points $(0, \leq 25 \% ; 1$, $26 \%-50 \% ; 2,51 \%-75 \% ; 3,76 \%-90 \%$; and $4,>90 \%$ stenosis), and then, the severity score was defined as the sum of scores of all segments. Coronary artery segments were defined as 29 segments according to the Coronary Artery Surgery Study (CASS) classification. All angiograms were evaluated by a single cardiologist (Y.M.), blinded to the clinical and laboratory data.

2.4. Statistical Analysis. Differences between 2 groups were evaluated by unpaired $t$-test for parametric variables, by Mann-Whitney $U$ test for nonparametric variables, and by chi-squared test for categorical variables. Differences among $\geq 3$ groups were evaluated by an analysis of variance with Scheffe's test for parametric variables, by Kruskal-Wallis test with Steel-Dwass test for nonparametric variables, and by chi-squared test for categorical variables. Correlations between plasma sestrin 2 or hsCRP levels and the severity of CAD were evaluated by Spearman's rank correlation test. To determine the cut-off point of plasma sestrin2 levels for $\mathrm{CAD}$, a receiver-operating characteristic curve was created, and then, the optimal cut-off point was determined to be $16.0 \mathrm{ng} / \mathrm{mL}$ as the point where the Youden index was maximum. Regarding the cut-off point of hsCRP levels, the previously reported cut-off point of $1.0 \mathrm{mg} / \mathrm{L}$ for CAD was used $[14,15]$. A forward stepwise multiple logistic regression analysis was performed to determine the independent association between sestrin 2 levels and CAD. All statistical analyses were carried out using the SPSS software package (IBM SPSS version 25, Tokyo, Japan). A $P$ value of $<0.05$ was considered to be statistically significant. The results are presented as the mean \pm SD or the median value.

\section{Results}

Among the 304 study patients, CAD was present in 175 patients (58\%) (1-vessel disease (1-VD), $n=73$; 2 -vessel disease (2-VD), $n=59$; and 3 -vessel disease (3-VD), $n=43$ ). Compared with 129 patients without CAD, 175 patients with $\mathrm{CAD}$ were older and had a male predominance; higher prevalence of hypertension, hyperlipidemia, and DM; and lower HDL-cholesterol levels (Table 1). Plasma hsCRP levels were higher in patients with $\mathrm{CAD}$ than in those without CAD (median 0.57 vs. $0.43 \mathrm{mg} / \mathrm{L}, P<0.01$ ). A stepwise increase in hsCRP levels was found depending on the number of $>50 \%$ stenotic coronary vessels: 0.43 in $\mathrm{CAD}(-), 0.54$ in 1$\mathrm{VD}, 0.57$ in $2-\mathrm{VD}$, and $0.60 \mathrm{mg} / \mathrm{L}$ in $3-\mathrm{VD} \quad(P<0.02)$ (Table 1). Notably, plasma sestrin2 levels were significantly higher in patients with CAD than in those without CAD (median 16.4 vs. $14.2 \mathrm{ng} / \mathrm{mL}, P<0.05$ ) (Figure 1). A stepwise increase in sestrin 2 levels was found depending on the number of $>50 \%$ stenotic vessels: 14.2 in $\mathrm{CAD}(-), 15.4$ in $1-\mathrm{VD}$, 17.3 in $2-\mathrm{VD}$, and $17.7 \mathrm{ng} / \mathrm{mL}$ in $3-\mathrm{VD}$ and was the highest in 3-VD $(P<0.05)$ (Figure 1). A high sestrin2 level $(>16.0 \mathrm{ng} / \mathrm{mL})$ was present in $38 \%$ of patients with $\mathrm{CAD}(-)$, $47 \%$ of $1-\mathrm{VD}, 66 \%$ of $2-\mathrm{VD}$, and $53 \%$ of $3-\mathrm{VD}(P<0.005)$. 


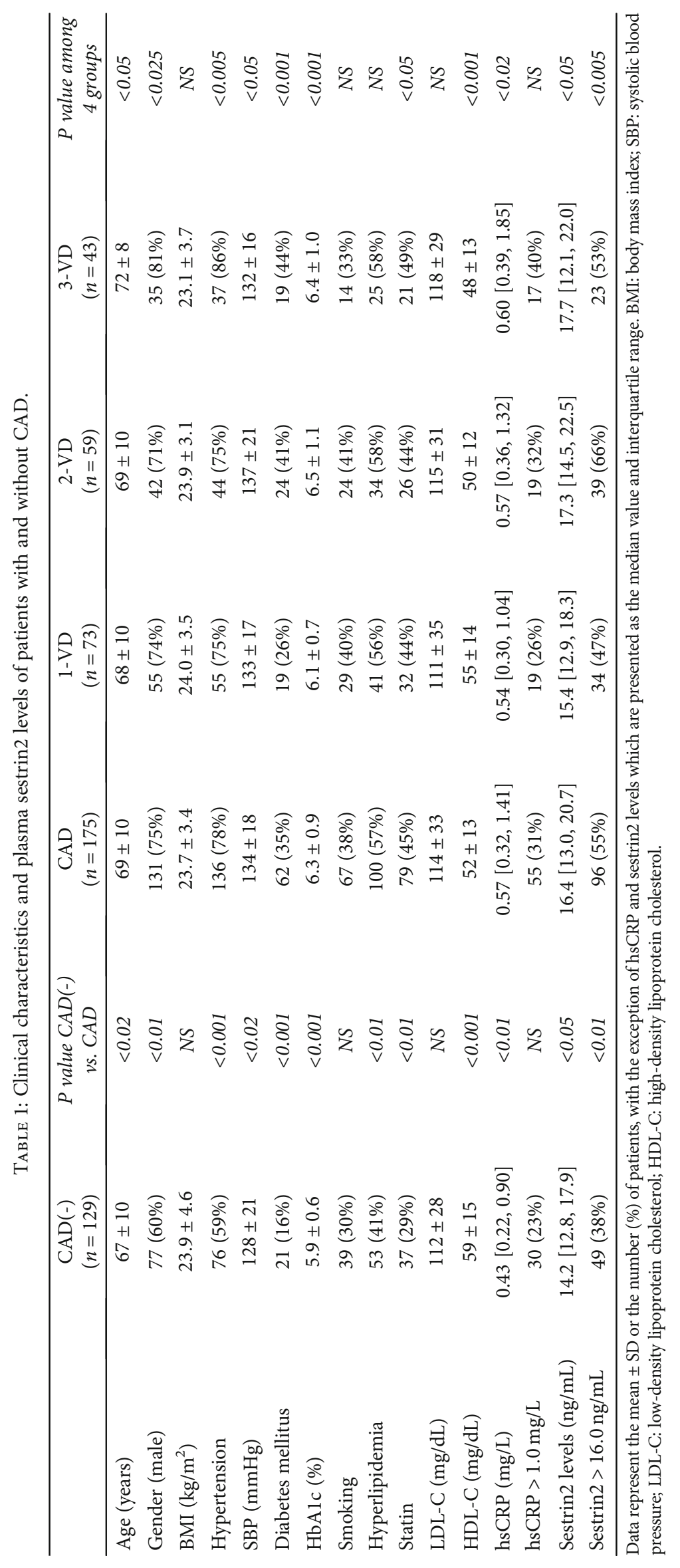



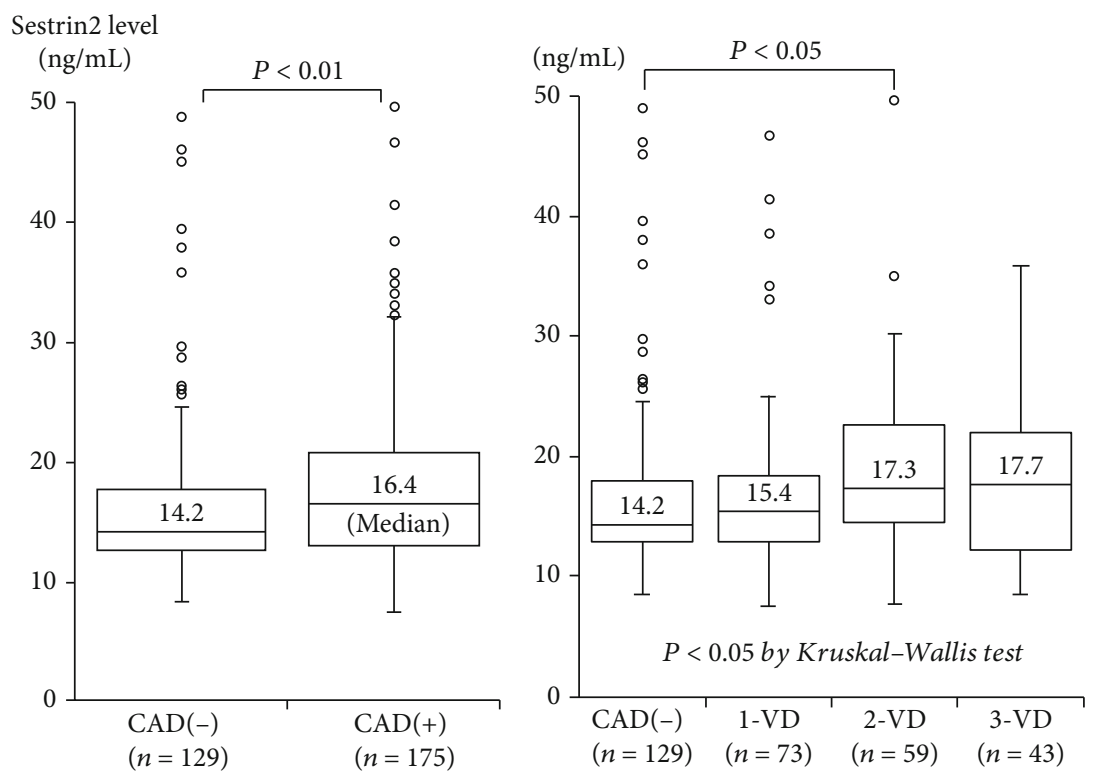

FIGURE 1: Plasma sestrin2 levels and the presence of CAD or the number of stenotic coronary vessels. Plasma sestrin2 levels were significantly higher in $\mathrm{CAD}$ than in $\mathrm{CAD}(-)$ (a). Moreover, sestrin2 levels in 4 groups of $\mathrm{CAD}(-), 1-\mathrm{VD}, 2-\mathrm{VD}$, and 3-VD were 14.2, 15.4, 17.3, and $17.7 \mathrm{ng} / \mathrm{mL}$, respectively, and were the highest in $3-\mathrm{VD}(P<0.05$ by Kruskal-Wallis test) (b). The central line represents the median, and the box represents the 25 th to 75 th percentiles. The whiskers represent the lowest and highest values in the 25th percentile minus 1.5 IQR and 75th percentile plus $1.5 \mathrm{IQR}$, respectively.

Although hsCRP levels correlated with the number of $>50 \%$ stenotic coronary segments and the severity score $(\mathrm{rs}=0.16$ and $\mathrm{rs}=0.16, P<0.01$ ), sestrin2 levels significantly, but weakly, correlated with the number of $>50 \%$ stenotic segments and the severity score ( $\mathrm{rs}=0.12$ and $\mathrm{rs}=0.13, P<$ 0.05).

Sestrin2 levels also correlated with age $(\mathrm{rs}=0.29, P<$ 0.001 ). To clarify the association between age and sestrin 2 levels, the 304 study patients were divided into 2 groups by age: patients aged $<70 \mathrm{yrs}(n=145)$ and $\geq 70 \mathrm{yrs}(n=159)$. Compared with patients aged $<70 \mathrm{yrs}$, those aged $\geq 70 \mathrm{yrs}$ had higher sestrin2 levels $(17.0$ vs. $14.2 \mathrm{ng} / \mathrm{mL}, P<0.001)$ and higher prevalence of CAD (65\% vs. $50 \%, P<0.025)$. Among patients aged $<70$ yrs, sestrin2 levels were significantly higher in patients with CAD than in those without CAD (15.4 vs. $13.6 \mathrm{ng} / \mathrm{mL}, P<0.05$ ), whereas among patients aged $\geq 70 \mathrm{yrs}$, sestrin 2 levels tended to be higher in patients with CAD than in those without CAD (17.2 vs. $15.9 \mathrm{ng} / \mathrm{mL})$ (Table 2).

To elucidate the independent association between plasma sestrin2 levels and CAD, variables (age, gender, hypertension, hyperlipidemia, statin use, DM, smoking, and HDLcholesterol, hsCRP, and sestrin2 levels) were entered into a multiple logistic regression model. Plasma sestrin2 levels were a significant factor associated with $\mathrm{CAD}$ independent of atherosclerotic risk factors. The odds ratio for CAD was $1.79(95 \% \mathrm{CI}=1.09-2.95)$ for the high sestrin2 level of $>16.0 \mathrm{ng} / \mathrm{mL}(P<0.025)$ (Table 3$)$.

\section{Discussion}

In the present study, plasma sestrin2 levels were significantly higher in patients with CAD than in those without CAD, and they positively, but weakly, correlated with the severity of CAD, defined as the numbers of $>50 \%$ stenotic vessels and segments and the severity score. High plasma sestrin2 levels were found to be a significant factor associated with CAD independent of atherosclerotic risk factors.

Regarding the association between sestrin 2 and atherosclerotic diseases, Xiao et al. [16] reported that sestrin2 expression was upregulated in aortic specimens from 12 patients with aortic dissection and that plasma sestrin2 levels were higher in 120 patients with aortic dissection than in 40 patients without dissection. In contrast, Chung et al. [17] investigated serum sestrin2 levels and carotid intima-media thickness (IMT) measured by ultrasonography in 194 diabetic patients. However, they found no significant correlation between serum sestrin2 levels and carotid mean IMT.

Regarding the association between sestrin2 levels and coronary atherosclerosis, Ye et al. [12] measured plasma sestrin2 levels in 119 patients with CAD (stable angina (SA), $n$ $=44$; unstable angina (UA), $n=41$; and acute myocardial infarction (AMI), $n=29$ ) and 35 without CAD. They reported sestrin2 levels to be higher in patients with CAD than in those without CAD and to be much higher in UA and AMI than in SA. However, in their study, any multivariate analysis was not performed in spite of some significant differences in atherosclerotic risk factors between patients with and without CAD. They also reported sestrin2 levels to correlate well with the Gensini score $(r=0.46)$, but this correlation coefficient was analyzed in CAD patients including patients with AMI. Since sestrin2 expression was demonstrated to be upregulated in cardiac macrophages of a mouse MI model [18], our present study excluded patients with AMI. In the present study, we found that plasma sestrin2 levels were significantly higher in 175 patients with CAD 
TABLE 2: Clinical characteristics and plasma sestrin2 levels in the subgroups of patients aged $<70$ years and $\geq 70$ years.

\begin{tabular}{|c|c|c|c|c|c|c|}
\hline & \multicolumn{3}{|c|}{ Age $<70$} & \multicolumn{3}{|c|}{ Age $\geq 70$} \\
\hline & $\begin{array}{l}\mathrm{CAD}(-) \\
(n=73)\end{array}$ & $C A D(-)$ vs. $C A D$ & $\begin{array}{c}\text { CAD } \\
(n=72)\end{array}$ & $\begin{array}{l}\mathrm{CAD}(-) \\
(n=56)\end{array}$ & $C A D(-)$ vs. $C A D$ & $\begin{array}{c}\text { CAD } \\
(n=101)\end{array}$ \\
\hline Age (years) & $60 \pm 8$ & NS & $60 \pm 7$ & $76 \pm 4$ & NS & $76 \pm 4$ \\
\hline Gender (male) & $45(62 \%)$ & $<0.025$ & $58(81 \%)$ & $32(57 \%)$ & NS & $73(71 \%)$ \\
\hline Hypertension & $39(53 \%)$ & $<0.01$ & $55(76 \%)$ & $37(66 \%)$ & NS & $81(79 \%)$ \\
\hline Diabetes mellitus & $13(18 \%)$ & $<0.025$ & $27(38 \%)$ & $8(14 \%)$ & $<0.025$ & $35(34 \%)$ \\
\hline Smoking & $26(36 \%)$ & NS & $33(46 \%)$ & $13(23 \%)$ & NS & $34(33 \%)$ \\
\hline Hyperlipidemia & $29(40 \%)$ & $<0.025$ & $43(60 \%)$ & $24(43 \%)$ & NS & $57(55 \%)$ \\
\hline Statin & $17(23 \%)$ & $<0.01$ & $33(46 \%)$ & $20(36 \%)$ & $<0.01$ & $46(45 \%)$ \\
\hline LDL-C (mg/dL) & $113 \pm 29$ & NS & $116 \pm 35$ & $110 \pm 27$ & NS & $113 \pm 31$ \\
\hline HDL-C (mg/dL) & $59 \pm 16$ & $<0.001$ & $50 \pm 12$ & $58 \pm 13$ & $<0.02$ & $53 \pm 14$ \\
\hline hsCRP (mg/L) & $0.40[0.21,0.94]$ & $<0.05$ & $0.58[0.30,1.52]$ & $0.46[0.26,0.87]$ & NS & $0.57[0.32,1.31]$ \\
\hline $\mathrm{hsCRP}>1.0 \mathrm{mg} / \mathrm{L}$ & $18(25 \%)$ & NS & $24(33 \%)$ & $12(21 \%)$ & NS & $31(30 \%)$ \\
\hline Sestrin2 levels $(\mathrm{ng} / \mathrm{mL})$ & $13.6[12.5,16.8]$ & $<0.05$ & $15.4[12.8,20.0]$ & $15.9[13.6,22.3]$ & NS & $17.2[13.3,21.4]$ \\
\hline Sestrin $2>16.0 \mathrm{ng} / \mathrm{mL}$ & $21(29 \%)$ & $<0.05$ & $34(47 \%)$ & $28(50 \%)$ & NS & $62(60 \%)$ \\
\hline
\end{tabular}

Data represent the mean $\pm \mathrm{SD}$ or the number (\%) of patients, with the exception of hsCRP and sestrin2 levels which are presented as the median value and interquartile range.

TABLE 3: Factors associated with CAD (multiple logistic regression analysis of the 304 study patients).

\begin{tabular}{lccc}
\hline & Odds ratio & $(95 \%$ CI $)$ & \\
\hline Male gender & 2.19 & $(1.25-3.83)$ & $<$ value \\
Hypertension & 1.88 & $(1.09-3.24)$ & $<0.01$ \\
Hyperlipidemia & 1.99 & $(1.17-3.40)$ & $<0.025$ \\
Diabetes mellitus & 2.15 & $(1.18-3.90)$ & $<0.02$ \\
High sestrin2 level $(>16.0 \mathrm{ng} / \mathrm{mL})$ & 1.79 & $(1.09-2.95)$ & $<0.02$ \\
\hline
\end{tabular}

The dependent variable was the presence of CAD. The analysis included age, gender, hypertension, hyperlipidemia, statin use, diabetes mellitus, smoking, and HDL-cholesterol $(<40 \mathrm{mg} / \mathrm{dL})$, hsCRP $(>1.0 \mathrm{mg} / \mathrm{L})$, and sestrin2 $(>16.0 \mathrm{ng} / \mathrm{mL})$ levels.

than in 129 without CAD and that they were a significant factor associated with CAD independent of atherosclerotic risk factors. We also found that sestrin 2 levels correlated with the severity of CAD. However, the correlation between sestrin2 levels and the severity of CAD, defined as the number of stenotic segments and the severity score, was significant but weak $(r=0.12$ and $r=0.13)$. Moreover, as shown in Figure 1, there was a substantial overlap in sestrin2 levels between patients with and without CAD. Therefore, plasma sestrin2 levels in patients with CAD may reflect not only the degree of coronary atherosclerosis but also that of atherosclerosis in other vascular beds, and sestrin2 levels are unlikely to be a good biomarker for the presence or severity of CAD. Since sestrin2 is considered to have antioxidant and anti-inflammatory properties [4, 7-9], high plasma levels of sestrin 2 in patients with CAD may reflect a compensatory response to increased oxidative stress and may be aimed at protecting against the progression of CAD. However, further studies are needed to elucidate the main source and role of high plasma sestrin2 levels in patients with CAD.

Sestrin 2 is suggested to be associated with aging and agerelated diseases $[10,11]$, and plasma sestrin2 levels were reported to positively correlate with age $[12,17]$. In our study, sestrin2 levels also correlated with age $(r=0.29)$.
Compared with patients aged $<70 \mathrm{yrs}$, those aged $\geq 70 \mathrm{yrs}$ more often had CAD and had higher sestrin2 levels. Moreover, in patients aged $<70 \mathrm{yrs}$, sestrin 2 levels were significantly higher in patients with CAD than in those without CAD. However, in patients aged $\geq 70$ yrs, sestrin2 levels tended to be higher in patients with CAD than in those without CAD.

Our study has several limitations. First, in our study, angiography was used to evaluate coronary atherosclerosis. Angiography cannot visualize plaques and only shows lumen characteristics. However, intravascular ultrasound (IVUS) or optical coherence tomography (OCT), which can visualize coronary plaques, was not always performed in our patients. Second, sestrin 2 was reported to be expressed and secreted by various cells, mainly by macrophages, $T$ lymphocytes, and endothelial cells [4-6]. However, since we did not measure sestrin 2 levels in the coronary sinus, our study did not provide any information about the main sources of sestrin 2 in patients with CAD. Third, our study was cross-sectional in nature and was unable to establish causality, since it only depicted some associations and proposed some hypotheses. Finally, as in our previous studies [19, 20], the present study was performed in Japanese patients undergoing coronary angiography, who are generally considered to be a highly 
selected population at high risk for CAD. Our results therefore may not be applicable to the general or other ethnic populations.

In conclusion, plasma sestrin2 levels in patients with $\mathrm{CAD}$ were found to be high and to be associated with the severity of CAD. Our results suggest that high sestrin 2 levels in patients with $\mathrm{CAD}$ may reflect a protective response against the progression of CAD.

\section{Data Availability}

The data that support the findings of this study are available from the corresponding author on reasonable request.

\section{Disclosure}

The sponsors had no role in the design, analysis, or interpretation of our study.

\section{Conflicts of Interest}

Our study has no conflicts of interest to disclose.

\section{Acknowledgments}

Financial funding was provided by Bayer Yakuhin Ltd, Daiichi Sankyo Company, and Pfizer Japan Inc.

\section{References}

[1] A. J. Kattoor, N. V. K. Pothineni, D. Palagiri, and J. L. Mehta, "Oxidative stress in atherosclerosis," Current Atherosclerosis Reports, vol. 19, no. 11, 2017.

[2] S. Steven, K. Frenis, M. Oelze et al., "Vascular inflammation and oxidative stress: major triggers for cardiovascular disease," Oxidative Medicine and Cellular Longevity, vol. 2019, no. 7092151, 26 pages, 2019.

[3] A. V. Budanov, T. Shoshani, A. Faerman et al., "Identification of a novel stress-responsive gene hi95 involved in regulation of cell viability," Oncogene, vol. 21, no. 39, pp. 6017-6031, 2002.

[4] H. J. Hu, Z. Y. Shi, X. L. Lin, S. M. Chen, Q. Y. Wang, and S. Y. Tang, "Upregulation of sestrin2 expression protects against macrophage apoptosis induced by oxidized low-density lipoprotein," DNA and Cell Biology, vol. 34, no. 4, pp. 296-302, 2015.

[5] M. G. Kim, J. H. Yang, K. M. Kim et al., "Regulation of toll-like receptor-mediated sestrin 2 induction by AP-1, Nrf2, and the ubiquitin-proteasome system in macrophages," Toxicological Sciences, vol. 144, no. 2, pp. 425-435, 2015.

[6] H. Wang, N. Li, X. Shao et al., "Increased plasma sestrin2 concentrations in patients with chronic heart failure and predicted the occurrence of major adverse cardiac events: a 36-month follow-up cohort study," Clinica Chimica Acta, vol. 495, pp. 338-344, 2019.

[7] L. Yi, F. Li, Y. Yong et al., "Upregulation of sestrin-2 expression protects against endothelial toxicity of angiotensin II," Cell Biology and Toxicology, vol. 30, no. 3, pp. 147-156, 2014.

[8] J. H. Yang, K. M. Kim, M. G. Kim et al., "Role of sestrin2 in the regulation of proinflammatory signaling in macrophages," Free Radical Biology \& Medicine, vol. 78, pp. 156-167, 2015.
[9] H. J. Hwang, T. W. Jung, J. H. Choi et al., "Knockdown of sestrin2 increases pro-inflammatory reactions and ER stress in the endothelium via an AMPK dependent mechanism," Biochimica et Biophysica Acta - Molecular Basis of Disease, vol. 1863, no. 6, pp. 1436-1444, 2017.

[10] Y. Sun, Y. Wu, S. Tang, H. Liu, and Y. Jiang, "Sestrin proteins in cardiovascular disease," Clinica Chimica Acta, vol. 508, pp. 43-46, 2020.

[11] W. Sun, Y. Wang, Y. Zheng, and N. Quan, "The emerging role of sestrin2 in cell metabolism, and cardiovascular and agerelated diseases," Aging And Disease, vol. 11, no. 1, pp. 154$163,2020$.

[12] J. Ye, M. Wang, Y. Xu et al., "Sestrins increase in patients with coronary artery disease and associate with the severity of coronary stenosis," Clinica Chimica Acta, vol. 472, no. 5, pp. 51-57, 2017.

[13] C. W. Hamm and E. Braunwald, "A classification of unstable angina revisited," Circulation, vol. 102, no. 1, pp. 118-122, 2000.

[14] H. Arima, M. Kubo, K. Yonemoto et al., "High-sensitivity Creactive protein and coronary heart disease in a general population of Japanese," Arteriosclerosis, Thrombosis, and Vascular Biology, vol. 28, no. 7, pp. 1385-1391, 2008.

[15] Y. Momiyama, A. Kawaguchi, I. Kajiwara et al., "Prognostic value of plasma high-sensitivity C-reactive protein levels in Japanese patients with stable coronary artery disease: the Japan NCVC-Collaborative Inflammation Cohort Study," Atherosclerosis, vol. 207, no. 1, pp. 272-276, 2009.

[16] T. Xiao, L. Zhang, Y. Huang et al., "Sestrin2 increases in aortas and plasma from aortic dissection patients and alleviates angiotensin II-induced smooth muscle cell apoptosis via the nrf2 pathway," Life Sciences, vol. 218, pp. 132-138, 2019.

[17] H. S. Chung, H. J. Hwang, S. Y. Hwang et al., "Association of serum sestrin2 level with metabolic risk factors in newly diagnosed drug-naïve type 2 diabetes," Diabetes Research and Clinical Practice, vol. 144, pp. 34-41, 2018.

[18] K. Yang, C. Xu, Y. Zhang, S. He, and D. Li, "Sestrin2 suppresses classically activated macrophages-mediated inflammatory response in myocardial infarction through inhibition of mTORC1 signaling," Frontiers in Immunology, vol. 8, 2017.

[19] S. Ibe, Y. Kishimoto, H. Niki et al., "Associations between plasma nesfatin-1 levels and the presence and severity of coronary artery disease," Heart and Vessels, vol. 34, no. 6, pp. 965970, 2019.

[20] M. Aoyama, Y. Kishimoto, E. Saita et al., "High plasma levels of soluble Talin-1 in patients with coronary artery disease," Disease Markers, vol. 2020, no. 2479830, 8 pages, 2020. 